\title{
Consulta de enfermagem na Atenção Primária à Saúde: cuidado às pessoas com doenças crônicas cardiometabólicas
}

\author{
Nursing Consultation in Primary Health Care: Care for people with chronic cardiometabolic diseases \\ Consulta de enfermería en la Atención Primaria de Salud: el cuidado a los individuos con enfermedades \\ crónicas cardiometabólicas
}

\section{Maria Clara Moreira Matias', Uiara Aline de Oliveira Kaizer ${ }^{\mathrm{II}}$, Thaís Moreira São-JoãoIII}

\begin{abstract}
Resumo: Objetivo: desenvolver um instrumento para Consulta de Enfermagem às pessoas com hipertensão arterial e/ou diabetes mellitus tipo 2 em seguimento na Atenção Primária à saúde. Método: estudo metodológico, composto por duas etapas, desenvolvido em Unidade Básica de Saúde (UBS) no interior do Estado de São Paulo. A Etapa 1 correspondeu à Construção do Instrumento e à Validação de Conteúdo por cinco juízes. A Etapa 2 correspondeu ao pré-teste e à avaliação de suas propriedades de medida. O questionário foi pré-testado com 30 pessoas com hipertensão arterial e/ou debates mellitus usuárias da UBS. Resultados: o questionário apresentou alto índice de validade de conteúdo (IVC $=0,96$ ) e moderada aplicabilidade (pelo tempo de aplicação). Conclusão: o instrumento se apresentou adequado à realidade e à necessidade da Atenção Primária em termos de conteúdo.

Descritores: Enfermagem; Diabetes mellitus; Hipertensão; Inquéritos e questionários; Estudos de validação
\end{abstract}

\begin{abstract}
Objective: to design a nursing consultation instrument for people with high blood pressure and/or type 2 diabetes mellitus being followed up in Primary Health Care. Method: methodological study comprising two stages developed in a Basic Health Unit (BHS) in the interior of the state of São Paulo. Stage 1 was related to designing the instrument and validating its content with five judges. Stage 2 was related to pre-testing and evaluation of its measurement properties. The questionnaire was pre-tested with 30 people with high blood pressure and/or diabetes mellitus that attended the UBS. Results: the questionnaire had a high content validity index (CVI $=0,96)$ and moderate feasibility (considering the administration time). Conclusion: the instrument was adequate to the reality and need of primary health care in terms of content.
\end{abstract}

Keywords: Nursing; Diabetes mellitus; High blood pressure; Surveys and questionnaires; Validation study

\footnotetext{
${ }^{\text {I }}$ Enfermeira. Residente em Cardiologia pela Universidade de São Paulo. São Paulo, São Paulo, Brasil. E-mail: mariacmmatias@gmail.com Orcid: https://orcid.org/0000-0003-2343-4349

II Enfermeira. Doutora pelo Programa de Pós-Graduação em Enfermagem da Faculdade de Enfermagem da Universidade Estadual de Campinas. Campinas, São Paulo, Brasil. E-mail: uiara_oliveira@hotmail.com Orcid: https://orcid.org/0000-0002-9115-8043

III Enfermeira. Docente da Faculdade de Enfermagem da Universidade Estadual de Campinas. Campinas, São Paulo, Brasil. E-mail: thaisms@gmail.com Orcid: https://orcid.org/0000-0002-8520-6483
} 
Resumen: Objetivo: desarrollar un instrumento para la Consulta de Enfermería a los individuos con hipertensión arterial y/o con diabetes mellitus tipo 2 en monitoreo en la Atención Primaria de Salud. Método: estudio metodológico dividido en dos etapas y llevado a cabo en una Unidad Básica de Salud (UBS) en el estado de São Paulo. La Etapa 1 consistió en la Elaboración del Instrumento y la Validez de Contenido por cinco expertos. La Etapa 2 correspondió a la prueba previa y a la evaluación de sus propiedades de medida. El cuestionario se aplicó previamente a 30 individuos con hipertensión arterial y/o con diabetes mellitus, todos usuarios de la UBS. Resultados: el cuestionario reveló alto índice de validez de contenido (IVC $=0,96$ ) y moderada aplicabilidad (por el tiempo de aplicación). Conclusión: el instrumento fue adecuado a la realidad y la necesidad de la Atención Primaria en cuanto al contenido.

Descriptores: Enfermería; Diabetes mellitus; Hipertensión; Encuestas y cuestionarios; Estudio de validación

\section{Introdução}

A hipertensão arterial (HA) e o diabetes mellitus tipo 2 (DM2) são consideradas doenças crônicas não transmissíveis (DCNT) e possuem considerável custo financeiro e de mão de obra para o Sistema Único de Saúde (SUS), além de serem de difícil enfrentamento e cuidado para a família e para o indivíduo afetado. Em 2015, os gastos do Brasil com o DM se aproximaram de $\mathrm{R} \$ 4$ bilhões, dada sua cronicidade, ou seja, o tratamento contínuo. ${ }^{1}$ Em 2018 , a HA foi responsável por $59 \%$ do custo direto relacionado ao tratamento medicamentoso; ou seja, ela representa relevante impacto financeiro (mais de $\mathrm{R} \$ 2$ bilhões por ano) no Brasil. ${ }^{2}$ Já os gastos estimados de doenças cardiovasculares aumentaram em 17\%, de 2010 a 2015, alcançando R $\$ 37,1$ bilhões no ano de $2015 .^{3}$

Diante do impacto que a HA tem no cenário em saúde é importante reconhecer que a doença se caracteriza por aumento e persistência dos níveis de pressão arterial (PA) sistólica $\geq$ 140 e diastólica $\geq 90 \mathrm{mmHg}$, causado principalmente pelo enrijecimento das artérias, e, consequentemente, por aumento da resistência vascular periférica (RVP), muitas vezes causado pelo alto consumo de sódio, sendo agravada por fatores de risco, como a obesidade. ${ }^{4}$

O DM2 corresponde a 90 a $95 \%$ de todos os casos de DM; por sua vez, é caracterizado por diminuição da secreção de insulina pelas células $\beta$ do pâncreas e/ou pela resistência à insulina e, 
consequentemente, pelo maior nível de glicose circulante na corrente sanguínea. É um parâmetro de positividade, para diagnóstico da doença, a taxa de glicose em jejum $\geq 126 \mathrm{mg} / \mathrm{dl}$ ou o percentual de hemoglobina glicada $\geq 6,5 \% .^{1}$

É possível assumir que as duas patologias (HA e DM) se interligam por meio de seus agravos e fatores de risco, somando-se a dislipidemia, síndrome metabólica, hereditariedade, má alimentação e significativo uso de sal, fumo, além da inatividade física estão ligados ao aumento de morte por causa cardíaca. ${ }^{5}$ Em 2016, a HA estava ligada diretamente a $45 \%$ das mortes por causas cardíacas, enquanto apenas $54,1 \%$ dos pacientes diagnosticados com HA a mantiveram controlada. ${ }^{6} \mathrm{O} \mathrm{DM}$, por sua vez, está presente na vida de 1 a cada 10 adultos norte-americanos e possui prevalência do tipo $2 .^{1}$

Em 2019, cerca de 17 milhões de pessoas foram acometidas por DM, sendo que o Brasil ocupava a quinta posição entre os países com maior número de afetados. Estima-se que, em 2045, mais de 20 milhões de pessoas terão DM, sendo que o Brasil registrará a metade das mortes decorrentes de DM estimadas para o conjunto de países da América do Sul e da América Central. ${ }^{7}$ Assim, evidencia-se a necessidade de maior conhecimento dos custos das DCNT, com vistas ao melhor investimento financeiro e pessoal na Atenção Primária à Saúde (APS) e objetivando a priorização das políticas integradas e intersetoriais de prevenção, enfrentamento e controle da hipertensão e do diabetes, visto que um tratamento preventivo traria maiores benefícios para o paciente e diminuiria os custos do SUS. ${ }^{2}$

Levando em consideração a cronicidade e a necessidade de tratamento contínuo da HA e do DM2 na APS, é preciso ressaltar o papel do enfermeiro. Por meio de intervenções e acompanhamento, ele está presente nas etapas de diagnóstico médico, adesão e compreensão do tratamento medicamentoso, prescrição e indicação de tratamentos não medicamentosos, atuação nos fatores de risco, acompanhamento de sinais e sintomas e intervenções nos aspectos sociais da pessoa - como contexto familiar em que vive e qual a rede de apoio que constitui. 
Consulta de enfermagem na Atenção Primária à Saúde: cuidado às pessoas com doenças crôn... $\mid 4$

Nessa perspectiva, um dos seus principais instrumentos de trabalho é a consulta de enfermagem (CE).

A CE é uma atividade privativa do enfermeiro, na qual é realizado o processo de enfermagem $(\mathrm{PE})$ em cinco etapas: levantamento de possíveis problemas de enfermagem e necessidades de saúde a se intervir, por meio de histórico e relato verbal; observação clínica de possíveis alterações, sinais e sintomas durante o exame físico (histórico de enfermagem); diagnóstico de enfermagem (DE), para estabelecimento de prioridades de cuidado; planejamento de intervenções e implementação; avaliação dos processos e seus resultados; e registro das atividades, sendo principalmente o DE e as condutas anexadas ao prontuário e ao histórico do paciente. Assim, por meio da CE, é possível que o enfermeiro atue junto a pessoa com HA e DM2, principalmente quanto à adesão aos tratamentos medicamentoso e não medicamentoso. ${ }^{8}$

Para se estabelecer um DE sistematizado, para melhor promover a ação e a assistência unificadas do enfermeiro, haja vista a premente necessidade dessa atuação em APS no SUS, destaca-se o uso da Classificação Internacional para a Prática de Enfermagem (CIPE). A CIPE foi proposta com vistas a unificar a nomenclatura utilizada pelos profissionais, o que seria um facilitador para o planejamento da assistência - por meio da organização dos dados do paciente - possibilitando uma classificação padronizada. ${ }^{9}$

Considerando a necessidade de assistência contínua, por conta da cronicidade da HA e do DM2, e considerando o papel do enfermeiro na prevenção de agravos e na promoção da saúde, o PE deve ser realizado de forma sistematizada e registrado adequadamente. Dessa maneira, é possível promover a melhor evolução e o acompanhamento da situação de saúde da pessoa com HA e DM - visto que são doenças silenciosas e que requerem diversos cuidados.

Assim, faz-se necessário um instrumento de DE para as pessoas com HA e DM2, a fim de facilitar e estimular a realização do PE na CE, buscando a melhor evolução e o adequado acompanhamento do seu estado de saúde. Portanto, este estudo teve como objetivo desenvolver 
5 | Matias MCM, Kaizer UAO, São-João TM

um instrumento para Consulta de Enfermagem às pessoas com HA e/ou DM2 em seguimento na Atenção Primária à Saúde.

\section{Método}

Trata-se de estudo com abordagem metodológica, ${ }^{10}$ para criação de instrumento para a CE na APS, composto por duas etapas e desenvolvido em uma Unidade Básica de Saúde (UBS) no interior do Estado de São Paulo. Essa UBS pertence à região sul de um distrito de saúde. Compõe a equipe de enfermagem: três enfermeiras e dez técnicos de enfermagem. A unidade constitui campo de atividade prática e estágio curricular para os discentes dos cursos de graduação em enfermagem e medicina. A população da área de abrangência da UBS é de 42.467 habitantes. Desses, 1.275 (3,0\%) são cadastrados com HA e 715 (1,7\%) com DM2. A comparação entre os dados locais da UBS e os parâmetros nacionais para DM e HA sugere fortemente a evidência de subnotificação, uma vez que parâmetros populacionais ${ }^{11}$ estabelecem que $21,4 \%$ da população maior de 18 anos apresentam HA e que 6,9\% têm DM - o que corresponderia, nesse cenário, a 9.088 habitantes e 2.930 habitantes, respectivamente.

A seguir, está descrito o percurso metodológico realizado.

\section{Etapa 1: Construção do Instrumento e Validação de Conteúdo}

Para construir um instrumento ou um questionário, é preconizado internacionalmente que o pesquisador defina as variáveis de interesse e suas dimensões, por meio de pesquisa bibliográfica, além de consulta a estudiosos da área e a representantes da população de interesse. ${ }^{12}$ Assim, foi realizado levantamento na literatura nacional e internacional, bem como levantamento documental junto a prontuários da UBS, a fim de levantar dados que permitam a construção de itens que avaliem de forma holística o paciente com HA e/ou DM2. 
Consulta de enfermagem na Atenção Primária à Saúde: cuidado às pessoas com doenças crôn... $\mid 6$

Quadro teórico

A Teoria do Autocuidado sinaliza a necessidade de intervenção quando há prejuízo no autocuidado de uma pessoa, ou seja, quando esta pessoa não progride sozinha na execução de medidas para o seu próprio bem-estar. Por meio do processo de enfermagem, a consulta deve direcionar o enfermeiro para observar e construir o cuidado, por meio de ações que visem o agir, o guiar, o apoiar, o ensinar e o manter-se junto à pessoa, sensibilizando-a sobre a manutenção do próprio bem-estar. ${ }^{13}$ Assim, a consulta de enfermagem guiada pelo instrumento em questão auxilia a organização do cuidado ofertado à pessoa hipertensa elou com diabetes, para que ela desenvolva o autocuidado em suas possibilidades pessoais, familiares e sociais, contando com a intervenção profissional.

Destaca-se que a consulta proposta atende ao Modelo de Atenção às Condições Crônicas, proposto pela Organização Pan-Americana da Saúde, ${ }^{12}$ que visa reorganizar a atuação na APS junto às pessoas com condições crônicas, adotando, como uma de suas premissas, o autocuidado apoiado. Nesse modelo, a consulta de enfermagem, guiada pelo autocuidado apoiado, orienta a prática assistencial - por meio do manejo clínico adequado da doença crônica, das mudanças necessárias no estilo de vida e da valorização de aspectos emocionais do paciente.

O referencial teórico selecionado para ser empregado nessa etapa foi a CIPE, desenvolvida pelo Conselho Internacional de Enfermeiros. ${ }^{9}$ A CIPE foi criada para permitir uma linguagem científica e unificada, comum à enfermagem mundial. Esta classificação permite ao enfermeiro identificar diagnósticos de enfermagem por meio de fenômenos de enfermagem. Validação de conteúdo

A validação de conteúdo é uma das etapas fundamentais para a elaboração de instrumentos e depende majoritariamente de uma avaliação por especialistas. Os especialistas ou juízes devem avaliar o instrumento como um todo, determinando sua abrangência (o quanto cada conceito foi coberto pelos itens e se todas as suas dimensões foram incluídas), sua clareza 
(redação dos itens e se foram redigidos de forma compreensível e adequada) e sua pertinência (ou representatividade - se os itens refletem os conceitos - e se são relevantes e adequados para os objetivos). ${ }^{14}$

Nessa fase, os especialistas podem sugerir a inclusão ou a eliminação de itens. A avaliação pelos juízes é composta por dois tipos distintos: avaliação qualitativa e avaliação quantitativa.

Para a avaliação quantitativa, foi usado o Índice de Validade de Conteúdo (IVC), que mede a proporção de juízes que estão em concordância sobre o instrumento e seus itens, sendo considerado aceitável um nível de concordância mínima de 0,80 (ou 80\%). ${ }^{1}$ Este método utiliza uma escala tipo Likert com pontuação de um a quatro. Assim, para cada item, as respostas incluem: 1 = não relevante ou não representativo, 2 = item necessita de grande revisão para ser representativo, 3 = item necessita de pequena revisão para ser representativo, $4=$ item relevante ou representativo. O índice é calculado por meio da soma de concordância dos itens que foram marcados por “ 3 ” ou “ 4 ” pelos juízes. Os itens que receberam pontuação “ 1 ” ou “2” devem ser revisados ou eliminados. Sua fórmula corresponde a: [IVC = Número de respostas “3” ou “4”/ Número total de respostas.

A avaliação qualitativa também pode ser realizada pelos juízes, por meio de sugestões de modificação ou revisão no texto ou no layout do documento, por exemplo. Assim, essa avaliação foi feita de forma individual e independente, pelos juízes, por meio de um processo interativo entre as pesquisadoras e os membros do comitê, no qual os juízes sugeriram quais pontos do instrumento poderiam ser melhorados a fim de clarificar os pontos controversos do instrumento final.

No presente estudo, foram selecionados cinco juízes, todos enfermeiros, que atenderam a pelo menos um dos seguintes critérios de seleção: ter experiência na APS e/ou ser pesquisador com conhecimento em desenvolvimento e validação de instrumentos. Os juízes, convidados por 
Consulta de enfermagem na Atenção Primária à Saúde: cuidado às pessoas com doenças crôn... $\mid 8$

e-mail, receberam uma versão do instrumento e cada juiz também recebeu um questionário específico para realizar a avaliação.

\section{Etapa 2: Pré-teste e avaliação das propriedades de medida}

Ao final da Etapa 1, a versão preliminar do instrumento foi submetida à etapa de prétestagem. Nesta etapa, o instrumento deve ser aplicado a um tamanho amostral que varia de trinta a quarenta sujeitos, de acordo com recomendações internacionais. ${ }^{15}$ Também nesta etapa, a praticabilidade do instrumento pode ser avaliada, neste caso, usando-se o tempo despendido no seu preenchimento, cronometrado em minutos. ${ }^{10}$

População, amostra e procedimento de amostragem

$\mathrm{Na}$ fase de construção do instrumento, não houve recrutamento de participantes; apenas na etapa 2 foram recrutadas pessoas com HA e/ou DM2, em seguimento clínico no referido serviço, que atenderam aos critérios de seleção: pessoas com diagnóstico médico prévio de HA e/ou DM2 e que eram capazes de estabelecer comunicação efetiva, demonstrando orientação e cognição aceitáveis para serem incluídas no estudo. Assim, para a etapa de pré-teste, foram incluídos, nesta pesquisa, 30 participantes.

Procedimento de coleta de dados

A coleta dos dados se deu no período de setembro de 2018 a fevereiro de 2019 e aconteceu apenas na etapa de pré-teste, com o instrumento já na versão final, após a sugestão dos juízes. Foi conduzida pela pesquisadora, de forma individual, em ambiente privativo, por meio de entrevista. A entrevista foi baseada nas recomendações para realização de consulta de enfermagem em Atenção Primária, com duração média de uma hora, a depender da necessidade do usuário. Nessa ocasião, também se deu a consulta ao prontuário de saúde, no referido campo de pesquisa.

Análise dos dados 
O IVC foi avaliado pelos cálculos já demonstrados, sendo considerado valor aceitável o IVC maior ou igual 0,80 . A praticabilidade foi avaliada pelo tempo despendido na entrevista, cronometrado em minutos.

Aspectos éticos

O projeto foi aprovado pelo Comitê de Ética em Pesquisa da instituição, sob Parecer 2.338.122/2018, em 19 de outubro de 2017, de acordo com a Resolução 466/2012, do Conselho Nacional de Saúde. Os participantes formalizaram sua anuência por meio da assinatura do Termo de Consentimento Livre e Esclarecido.

\section{Resultados}

A 1a. etapa consistiu na criação e na validação do conteúdo do instrumento. O processo de criação se baseou na consulta às literaturas nacional e internacional - quanto aos principais aspectos a serem avaliados durante uma CE e quanto aos fenômenos de saúde mais observados nos pacientes em seguimento.

Após a criação do instrumento, ele foi submetido à apreciação de cinco juízes quanto aos aspectos de pertinência e clareza dos itens. O comitê de juízes foi composto por cinco enfermeiras, descritas a seguir: uma enfermeira com ampla experiência em atendimento ambulatorial em DM, doutoranda; duas enfermeiras da Estratégia da Saúde da Família, sendo uma mestra e uma doutoranda; uma enfermeira atuante na gestão do serviço; e uma enfermeira docente em enfermagem, com ampla experiência em manejo e pesquisas envolvendo a CIPE.

Foram levantados os principais DE elencados em consultas de enfermagem na UBS nos meses de janeiro de 2017 a fevereiro de 2018. Durante o levantamento de DE junto aos 30 prontuários das pessoas selecionadas, que estavam em seguimento na Atenção Primária na UBS em apreço, foi notada a ausência do registro das etapas de diagnóstico e planejamento do PE, mesmo tendo sido realizada a CE. 
O quadro 1 demonstra os DE selecionados. Destaca-se que, com a confluência das doenças - HA e DM2 - os DE encontrados foram observados tanto em pessoas com DM2, como nas com HA e também naquelas que apresentava as doenças simultaneamente - o que é considerado frequente, por sua cronicidade, suas similaridades e seus fatores de risco.

Quadro 1 - Diagnósticos de enfermagem para pessoas com HA e DM selecionadas e incluídas na Classificação Internacional para a Prática de Enfermagem (CIPE) 2017.

\begin{tabular}{|c|c|}
\hline Abuso de álcool (ou alcoolismo) & Hipoglicemia \\
\hline Abuso de tabaco (ou de fumo) & Ingestão de alimentos prejudicada \\
\hline Adesão ao regime terapêutico eficaz & Integridade da pele eficaz \\
\hline Adesão ao regime terapêutico prejudicado & Nível de glicose sanguínea nos limites normais \\
\hline Apoio familiar positivo & Percepção tátil prejudicada \\
\hline Baixo conhecimento sobre o regime de terapêutico & Polifármacos (ou polifarmácia) \\
\hline Comportamento de busca de saúde & Pressão arterial alterada \\
\hline Comportamento de exercício físico prejudicado & Pressão arterial nos limites normais \\
\hline Condição psicológica prejudicada & Problema com a aquisição da medicação \\
\hline Dispneia & Resposta à terapia eficaz \\
\hline Dor isquêmica & Risco de função cardíaca prejudicada \\
\hline Dor neurogênica & Risco de função neurovascular periférica prejudicada \\
\hline Dor vascular & Risco de úlcera em pé ou perna \\
\hline Edema periférico & Sobrepeso \\
\hline Efeito colateral da medicação & Tabagismo pregresso \\
\hline Falta de apoio familiar & Úlcera em pé ou perna \\
\hline Hiperglicemia & Visão prejudicada \\
\hline
\end{tabular}


Após a criação do instrumento, a apreciação dos juízes apresentou um IVC de 0,96. O instrumento preliminarmente construído possuía páginas frente e verso (para coleta de dados) e outra página, com os diagnósticos e o planejamento de enfermagem, os quais abrangiam os principais dados a serem coletados e os aspectos a serem avaliados. Os itens compreendiam dados de caracterização sociodemográfica e clínica, hábitos e estilo de vida, exame físico e dados laboratoriais.

Os juízes dispuseram de 15 dias para fazer suas considerações sobre o instrumento, de forma individual e remota. Ao final desse período, enviaram suas avaliações por e-mail. A equipe de pesquisa reuniu-se, a fim de acatar as sugestões e obter a versão final. Ao final da Etapa 1, obteve-se a versão final do instrumento (Apêndice 1).

Assim, após as duas etapas, foi formado um instrumento com três laudas: duas delas destinadas à coleta estruturada de dados e uma destinada aos diagnósticos e ao planejamento de enfermagem. O instrumento final foi submetido ao pré-teste, pela pesquisadora, para avaliação de sua praticabilidade, a uma amostra de 30 pessoas com HA e/ou DM2 que frequentavam a UBS. Nessa ocasião, as pessoas foram informadas de que participariam de uma etapa de uma pesquisa e, as que quiseram participar, assinaram o TCLE. No que tange à praticabilidade do instrumento, observou-se que a média de tempo dispensado para sua aplicação foi de aproximadamente 50 minutos.

\section{Discussão}

A enfermagem, enquanto ciência utiliza-se de um corpo de conhecimento exclusivo e afirma-se como uma disciplina, utilizando o pensamento teórico num percurso de grande evolução conceitual, baseando-se em teorias com valores próprios para a profissão. ${ }^{13}$ Para tal, utiliza-se da CE que, além de ser uma importante ferramenta de educação em saúde, propicia 
um vínculo entre o profissional e a pessoa que utiliza o serviço, além de possibilitar o desenvolvimento da autonomia e da independência profissional. ${ }^{16}$

Para isso, a utilização de padronização é necessária, com vistas à criação e à validação de instrumentos com propriedades de medida válidas. Dessa maneira, buscou-se a validação de conteúdo do instrumento de CE aplicado à pessoa com DM2 e/ou HA. Essa validade foi alcançada pela concordância entre os juízes, representada pelo valor de $\mathrm{IVC}=0,96$. O instrumento foi considerado adequado em relação aquilo que ele se propõe a medir, já que, para se verificar a validade de novos instrumentos, a literatura sugere e utiliza, frequentemente, o valor de IVC $=0,80$ para o instrumento no geral..$^{14}$

Durante o processo de validade de conteúdo do instrumento, as diferentes experiências e conhecimentos profissionais do comitê de especialistas mostraram-se oportunas, pois diferentes saberes teórico-práticos foram agregados para avaliação das pessoas com DM2 e/ou HA. Outra contribuição que se destaca em estudos de validação é a possibilidade de validar um instrumento de acordo com a demanda, atendendo às necessidades de saúde da população e sendo importante para o cuidado de enfermagem. ${ }^{16}$

Os aspectos a serem avaliados em uma pessoa com HA e/ou DM2 em seguimento na APS são diversos e se referem, em boa parte, aos comportamentos relacionados à saúde, como a medida ambulatorial e residencial da pressão arterial e/ou da glicemia capilar; a adesão e manutenção de uma dieta saudável, hipossódica e/ou com baixo consumo de carboidratos; a prática regular de atividade física; o comparecimento a consultas médicas e de enfermagem; a adesão ao tratamento medicamentoso; o monitoramento dos sintomas relacionados à descompensação das doenças; a manutenção ou a perda ponderal de peso, quando indicada; dentre outros. ${ }^{17}$ Entretanto, é conhecida a necessidade de sintetização de uma coleta de dados durante uma CE, para melhor direcionamento dos dados a serem coletados e, posteriormente, avaliados pelo enfermeiro - inclusive por conta da otimização do tempo de trabalho. 
Durante o levantamento de DE em prontuários de pessoas com DM2 e/ou HA em seguimento na APS, notou-se a ausência do registro das etapas de diagnósticos e planejamento do PE. Essa percepção corrobora os achados de outro estudo, realizado no mesmo município, em que a ausência da execução e do registro é atribuída a fatores extrínsecos, como a falta de recursos humanos, a falta de estrutura física para atendimento e a própria cultura institucional na qual o enfermeiro responsável não possui oportunidade de delimitação de quantidade de pessoas a serem acompanhadas, seja pela própria CE ou pelo acolhimento, por exemplo. ${ }^{18}$

O desvio de função, a falta de espaço físico, o trabalho multifacetado do enfermeiro e a burocratização do cuidado são elementos deteriorantes. ${ }^{15} \mathrm{Um}$ estudo discutiu as fragilidades e os aspectos limitantes para a execução da CE no contexto da Estratégia Saúde da Família de um distrito sanitário do Sul do país e, apesar do reconhecimento do papel da consulta na práxis dos enfermeiros, ainda se verificam entraves para sua execução como a falta de incentivo para realização, a falta de compreensão acerca da importância da CE para a melhor execução do trabalho do enfermeiro e a falta de apropriação do processo de enfermagem. ${ }^{16,18}$

A etapa de coleta de dados do instrumento elaborado auxilia no conhecimento mais aprofundado da situação econômica e social do indivíduo, uma vez que, após seu preenchimento e sua indexação ao prontuário, possibilita a redução de dados a serem levantados em acompanhamentos seguintes. Além disso, tal etapa do instrumento se torna um guia dos aspectos mais relevantes a serem observados pelo enfermeiro, mas não inibe a realização de anamnese e exame físico guiados conforme a demanda conhecida pelo profissional.

Já a segunda parte do instrumento, compreendida pelo conjunto de Diagnósticos e Planejamento de Enfermagem, pode ser utilizada independentemente da forma de coleta de dados elencada, facilitando a execução do processo de enfermagem de forma sistematizada e conforme a taxonomia da CIPE. ${ }^{9}$ Além disso, possui espaço aberto para a inclusão de outros 
diagnósticos, além dos pré-selecionados e validados para tal grupo, e também para definição de resultados e intervenções conforme a realidade compreendida pelo enfermeiro.

Mesmo com a sintetização de diversos aspectos, nesta pesquisa, obteve-se um instrumento considerado extenso, com tempo médio de aplicação de 50 minutos. Porém, há de se considerar que boa parte das informações não serão coletadas em todas as consultas, por exemplo, a caracterização sociodemográfica e clínica, que poderá ser preenchida alterando alguns dados ou incluindo informações da condição de saúde pré-existentes, o que promoveria redução desse tempo. Também a CE teria uma periodicidade e o enfermeiro poderia agendar essa avaliação conforme a disponibilidade do tempo de trabalho e levando em conta a demanda e a prioridade das pessoas, conforme grau de avaliação.

A questão do tempo dispendido para a execução e o registro do PE sugere ser um fator limitante para sua execução. Assim, a praticabilidade do instrumento pode ser considerada moderada em relação à realidade de trabalho dos enfermeiros atuantes na APS - o que pode ser entendido como uma limitação para sua aplicabilidade. A literatura aponta que a quantidade de atividades administrativas desenvolvidas pelos enfermeiros parece ser um entrave para realização de atividades assistenciais e privativas, como a CE. ${ }^{19}$

Entretanto, a forma de construção do instrumento permitiu a utilização adequada às necessidades de atendimento do profissional, pois, por meio da CE, o enfermeiro consegue maior resolutividade dos problemas da população, favorecendo a qualidade do cuidado prestado por ele, que se torna, muitas vezes, o profissional de referência para as pessoas na APS. ${ }^{16}$ Vale destacar a utilização da Teoria do Autocuidado durante essas etapas, sendo que, para utilização de qualquer teoria de enfermagem, sobretudo as grandes teorias, é necessário compreender a natureza das pessoas e a sua interação com o meio em que vivem, a fim de ajudar a definir quais as intervenções que melhoram sua saúde e seu bem-estar. 
A melhoria do cuidado deve ser o principal objetivo e tem-se revelado útil para a expansão da profissão enquanto ciência. ${ }^{12}$ Além disso, a integralidade do cuidado, pautado na assistência sistematizada e com toda fundamentação teórica, é essencial para o enfermeiro.

\section{Conclusão}

Conclui-se que o instrumento para CE na APS a pessoas com DM2 e HA tem validade de conteúdo e praticabilidade. Após todas as etapas de construção e validação, o instrumento construído se mostrou efetivo para sua utilização na APS, pelo seu conteúdo coerente com a realidade e a necessidade de saúde, elencando os principais aspectos a serem visualizados pelo enfermeiro e facilitando o processo de raciocínio clínico no levantamento dos diagnósticos, dos resultados e das ações de enfermagem.

No pré-teste, o instrumento também se mostrou adequado para auxílio na evolução e na avaliação do estado de saúde posterior da pessoa com DM2 ou HA, o que sugere sua utilização para um mais acurado seguimento de saúde da população. O instrumento proposto constitui uma ferramenta que pode ser reproduzida nos diversos serviços de saúde que prestam atendimento às pessoas com essas condições de saúde e pode também ajudar na organização do processo de trabalho dos enfermeiros e na evolução da categoria enquanto ciência.

Destaca-se como limitação desta pesquisa a subjetividade da avaliação do comitê de juízes, sendo necessárias outras aplicações de validade adicionais, como a validação estatística dos parâmetros de praticabilidade e reprodutibilidade, para maiores consistência e representatividade na prática assistencial de enfermagem. Novas pesquisas são recomendadas, com maior tamanho amostral, com vistas a demonstrar a robustez do instrumento, que pode ser aplicado a outros contextos de doenças crônicas. Estratégias como essa são de baixo custo, podendo ser associadas ao cuidado usual e implementadas no atendimento à pessoa com DM ou 
HA. O enfoque no autoconhecimento e no autocuidado pode se constituir em ferramenta eficaz para a construção do vínculo do usuário com a unidade e para o estímulo à sua autonomia.

\section{Referências}

1. Sociedade Brasileira de Diabetes. Diretrizes: Sociedade Brasileira de Diabetes 2019-2020 [Internet]. São Paulo: Clannad; 2020 [acesso em 2020 jul 15]. Disponível em: https://www.diabetes.org.br/profissionais/images/DIRETRIZES-COMPLETA-2019-2020.pdf

2. Nilson EAF, Andrade RCS, Brito DA, Oliveira ML. Costs attributable to obesity, hypertension, and diabetes in the Unified Health System, Brazil. Rev Panam Salud Publica. 2020;44:e32. doi: 10.26633/RPSP.2020.32

3. Siqueira ASE, Siqueira-Filho AG, Land MGP. Analysis of the economic impact of cardiovascular diseases in the last five years in Brazil. Arq Bras Cardiol. 2017;109(1):39-46. doi: 10.5935/abc.20170068

4. Malachias MVB, Gomes MAM, Nobre F, Alessi A, Feitosa AD, Coelho EB. $7^{\text {a }}$ Diretriz brasileira de hipertensão arterial: capítulo 2 - diagnóstico e classificação. Arq Bras Cardiol. 2016;107(3):7-13. doi: 10.5935/abc.20160152

5. American Heart Association. Heart Disease and stroke statistics-2020 update: a report from the American Heart Association. Circulation. 2020;141:e139-e596. doi: 10.1161/CIR.0000000000000757

6. Sociedade Brasileira de Cardiologia. $7^{\mathbf{a}}$ Diretriz Brasileira de Hipertensão Arterial. Arq Bras Cardiol [Internet]. 2016 [acesso em 2018 fev 01];107(3). Disponível em http://publicacoes.cardiol.br/2014/diretrizes/2016/05_HIPERTENSAO_ARTERIAL.pdf

7. International Diabetes Federation (IDF). IDF Diabetes Atlas [Internet]. 9th ed. Brussels, Belgium: IDF; 2019 [cited 2020 Apr 25]. Available from: http://www.diabetesatlas.org

8. CONSELHO FEDERAL DE ENFERMAGEM. Resolução no 358, de 15 de outubro de 2009. Dispõe sobre a sistematização da assistência de enfermagem. Brasília, DF: COFEN, 2009. Disponível em: http://www.cofen.gov.br/resoluo-cofen-3582009_4384.html. Acesso em: 01 fev. 2018.

9. Garcia TR, organizador. Classificação Internacional para a Prática de Enfermagem $\left(\mathrm{CIPE}^{\circledR}\right)$ : versão 2015. Porto Alegre: Artmed; 2016.

10. Polit DF, Beck CT. Nursing research: generating and assessing evidence for nursing practice. 
Philadelphia: Wolters Kluwer; 2016.

11. Instituto Brasileiro de Geografia e Estatística (IBGE). Síntese de indicadores sociais: uma análise das condições de vida da população brasileira: 2016. Rio de Janeiro: IBGE; 2016. 146 p. (Estudos e Pesquisas. Informação Demográfica e Socioeconômica; 36)

12. Mendes EV. O cuidado das condições crônicas na atenção primária à saúde: o imperativo da consolidação da estratégia da saúde da família [Internet]. Brasília (DF): Organização Pan-Americana da Saúde; $2012 \quad$ [cited $2015 \quad$ Jun 10$]. \quad 512 \quad$ p. $\quad$ Available from: https://bvsms.saude.gov.br/bvs/publicacoes/cuidado_condicoes_atencao_primaria_saude.pdf

13. Queirós PJP, Vidinha TSS, Almeida Filho AJ. Autocuidado: o contributo teórico de Orem para a disciplina e profissão de Enfermagem. Referência. 2014;4(3):157-64. doi: 10.12707/RIV14081

14. Coluci MZO, Alexandre NMC, Milani D. Construção de instrumentos de medida na área da saúde. Ciênc Saúde Colet. 2015;20(3):925-36. doi: https://doi.org/10.1590/1413-81232015203.04332013

15. Lidwine LB, Prinsen CAC, Bouter LM, de Vet HCW, Terwee CB. The COnsensus-based Standards for the selection of health Measurement INstruments (COSMIN) and how to select an outcome measurement instrument. Braz J Phys Ther. 2016;20(2):105-13. doi: 10.1590/bjpt-rbf.2014.0143

16. Silva KM, Santos SMA. A consulta de enfermagem na estratégia de saúde da família: realidade de um distrito sanitário. Rev Enferm UFSM. 2016;6(2):248-58. doi: 10.5902/2179769218079

17. Silveira LCJ, Rabelo-Silva ER, Ávila CW, Moreira LB, Dickson VVD, Riegel B. Cross-cultural adaptation of the self-care of hypertension inventory into Brazilian Portuguese. J Cardiovasc Nurs. 2018;33(3):289-95. doi: 10.1097/JCN.0000000000000442

18. Moraes JT, Fonseca DF, Mata LRF, Oliveira PP, Sampaio FC, Silva JF. Validação de um instrumento para consulta de enfermagem à pessoa com diabetes mellitus e/ou hipertensão arterial. Referência. 2018; $\operatorname{serIV(19):127-35.~doi:~10.12707/RIV18041~}$

19. Spazapan MP. Processo de enfermagem na atenção primária: percepção de enfermeiros de CampinasSP [dissertação]. Campinas: Faculdade de Enfermagem da Universidade Estadual de Campinas; 2017. 92 p. 
Apêndice 1 - Instrumento final para consulta de enfermagem às pessoas com hipertensão arterial e/ou diabetes mellitus tipo 2 na atenção primária - versão apresentada aos juízes. Campinas, 2018. 
INSTRUMENTO PARA CONSULTA DE ENFERMAGEM

NA HIPERTENSÃO ARTERIAL E/OU DIABETES MELLITUS TIPO 2 NA ATENÇÃO PRIMÁRIA

1. COLETA DE DADOS

Nome: 1.1 CARACTERIZAÇÃO SOCIODEMOGRÁFICA

Nome da mãe:

Cartão SUS:

Unidade de Saúde:

Endereço:

Com quem mora/composição familiar:

Situação conjugal: $\square$ solteiro(a) $\square$ casado(a)/vive com companheiro(a) $\square$ viúvo(a) $\square$ separado(a)/divorciado(a)

Escolaridade: $\square \mathrm{EF}$ incompleto $\square \mathrm{EF}$ completo $\square \mathrm{EM}$ incompleto $\square \mathrm{EM}$ completo $\square \mathrm{ES}$ incompleto $\square \mathrm{ES}$ completo

Características da moradia: $\square$ própria $\square$ alugada $\square$ cedida $\square$ ocupação

Número de cômodos:

$\square$ alvenaria $\square$ madeira $\square$ mista $\square$ plástico/lona $\square$ outro:

Ocupação:

Religião:

Renda familiar (R\$): Situação de saneamento:

Rede de apoio (com quem vive/convive, pessoas com quem pode contar no dia a dia):

\subsection{CARACTERIZAÇẢO CLÍNICA}

Antecedentes Familiares: $\square$ IAM. Quem:

$\square$ AVE. Quem:

$\square$ Morte súbita. Quem:

$\square$ Outras. Quais e quem:

$\square$ DM. Quem:

$\square$ HAS. Quem:

Hipóteses Diagnósticas: $\square$ DM $\square$ HAS $\square$ Dislipidemia $\square$ Outras:

Medicamentos em uso:

\begin{tabular}{|c|c|c|}
\hline Princípio ativo & Dose & Horários \\
\hline & & \\
\hline & & \\
\hline & & \\
\hline & & \\
\hline & & \\
\hline & & \\
\hline & & \\
\hline & & \\
\hline
\end{tabular}

Dificuldades para adesão medicamentosa:

1.3 HÁBITOS E ESTILO DE VIDA

Quantas pessoas realizam as refeiçőes diariamente na casa:

Quem é o (a) responsável pelo preparo dos alimentos:

-Café da manhã:

-Lanche da manhã:

-Almoço:

-Café da tarde:

- Jantar:

Lanche da noite:

Litros de óleo/semana: __ Kg de sal/mês:

Uso de temperos dos prontos (caldo em tabletes/sachês): $\square$ Não $\square$ Sim. Frequência:

Consumo de alimentos processados e ultra processados:

Dificuldades para adesão alimentar:

Ingesta Hídrica (copos de água/dia):

Consumo de álcool: $\square$ Nega $\square$ Atual. Tipo de bebida:

$\square$ Pregresso. Tipo de bebida:

Prática de Atividade Física: $\square$ AF de Lazer. Tipo:

AF de Locomoção. Tipo

AF no Trabalho. Tipo:

Câncer. Quem:

DN:

Equipe de Referência: 


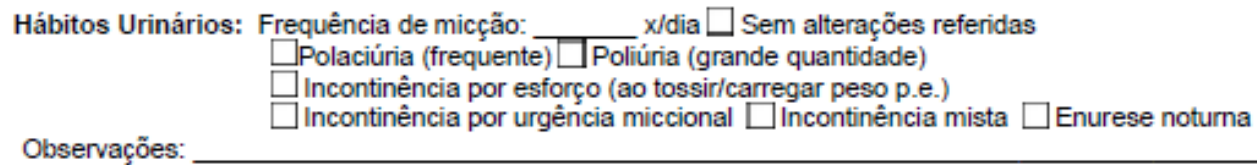

Hábitos Intestinais: Frequência de evacuação: __ x/semana $\square$ Sem alterações referidas

$\begin{array}{ll} & \square \text { Incontinência Fecal } \square \text { Sangue nas fezes } \\ \text { Observações: } & \square \text { Constipação Frequente } \square \text { Força excessiva para evacuar } \\ \text { Sono e Repouso: } \square \text { Sem alterações referidas } \square \text { Dificuldades para adormecer } \\ \text { Observações: }\end{array}$

Acuidade visual: Úlitima avaliação do oftalmologista: __________ $\square$ Sem alterações referidas $\square$ Uso de óculos Observações:

Tabagismo:

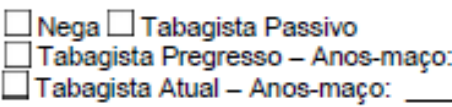

\subsection{EXAME FÍSICO}

Peso: kg Altura: $\mathrm{cm}$ Circunferência Abdominal: $\mathrm{cm}$ IMC $\mathrm{kg} / \mathrm{m}^{2}$

Adultos (20-59 anos): $\square<18,5$ (baixo peso) $\square 18,5$ - 24,9 (eutrofia) $\square 25-29,9$ (sobrepeso) $\square 30$ - 39,9 (obesidade) Idosos ( 260 anos): $\quad \square<22$ (baixo peso) $\square 22-27$ (eutrofia) $\square>27$ (excesso de peso)

PA sentado: $\mathrm{mmHg}$ PA em pé: $\mathrm{mmHg} \mathrm{FC:}$ bpm FR: $\mathrm{rpm}$

Pulso Carotídeo (D) $\square$ Cheio $\square$ Filforme $\square$ Ritmico $\square$ Amítmico Pulso Carotídeo (E): $\square$ Cheio $\square$ Filiforme $\square$ Rítmico $\square$ Arrítmico Pulso Radial (D): $\square$ Cheio $\square$ Filforme $\square$ Rítmico $\square$ Arritmico Pulso Radial (E): $\square$ Cheio $\square$ Filiforme $\square$ Rítmico $\square$ Arritmico

Ausculta cardiaca: Ausculta pulmonar:

Avaliação de MMII: $\square$ Veias varicosas $\square$ Edema $\left(\_/ 4+\right) \square$ Dor (caracterizar:

$\square$ Pele brilhante $\square$ Ausência de pelos $\square$ Coloração (caracterizar:

$\square$ Temperatura elevada $\square$ Temperatura diminuída

—esão (caracterizar:

Avaliação dos pés: $\square$ Sensibilidade Tátil Preservada $\square$ Alteração de sensibilidade tátil $\square$ Calosidades $\square$ Lesão

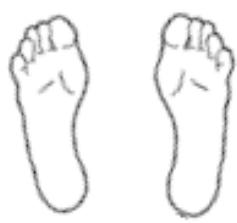

Observações:

Avaliação das unhas: $\square$ Preservadas $\square$ Pontiagudas $\square$ Encravadas $\square$ Outro: Avaliação das articulações: $\square$ Amplitude preservada $\square$ Amplitude limitada $\square$ Edema $\square$ Dor $\square$ Outro:

Pulso pedioso (D): $\square$ Ausente $\square$ Presente $\square$ Cheio $\square$ Filiforme $\square$ Rítmico $\square$ Arrítmico Pulso pedioso (E): $\square$ Ausente $\square$ Presente $\square$ Cheio $\square$ Filiforme $\square$ Rítmico $\square$ Arrítmico

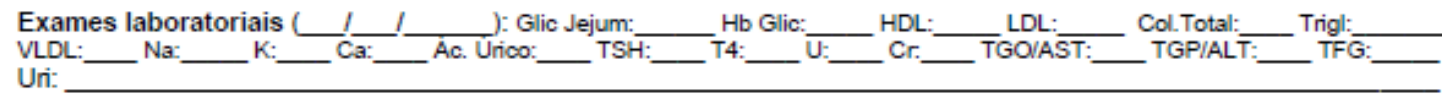

Outras observações: 
2. DIAGNÓSTICOS DE ENFERMAGEM - CIPE (Enumere para correlação entre os itens 2, 3.1 e 3.2)

Abuso de Álcool (ou Alcoolismo)
Abuso de Tabaco (ou de Fumo)
Adesäo ao Regime Terapêutico Eficazz
Adesäo ao Regime Terapêutico Prejudicado
Apoio Familiar Positivo
Baixo Conhecimento Sobre o Regime Terapêutico

3. PLANEJAMENTO DE ENFERMAGEM
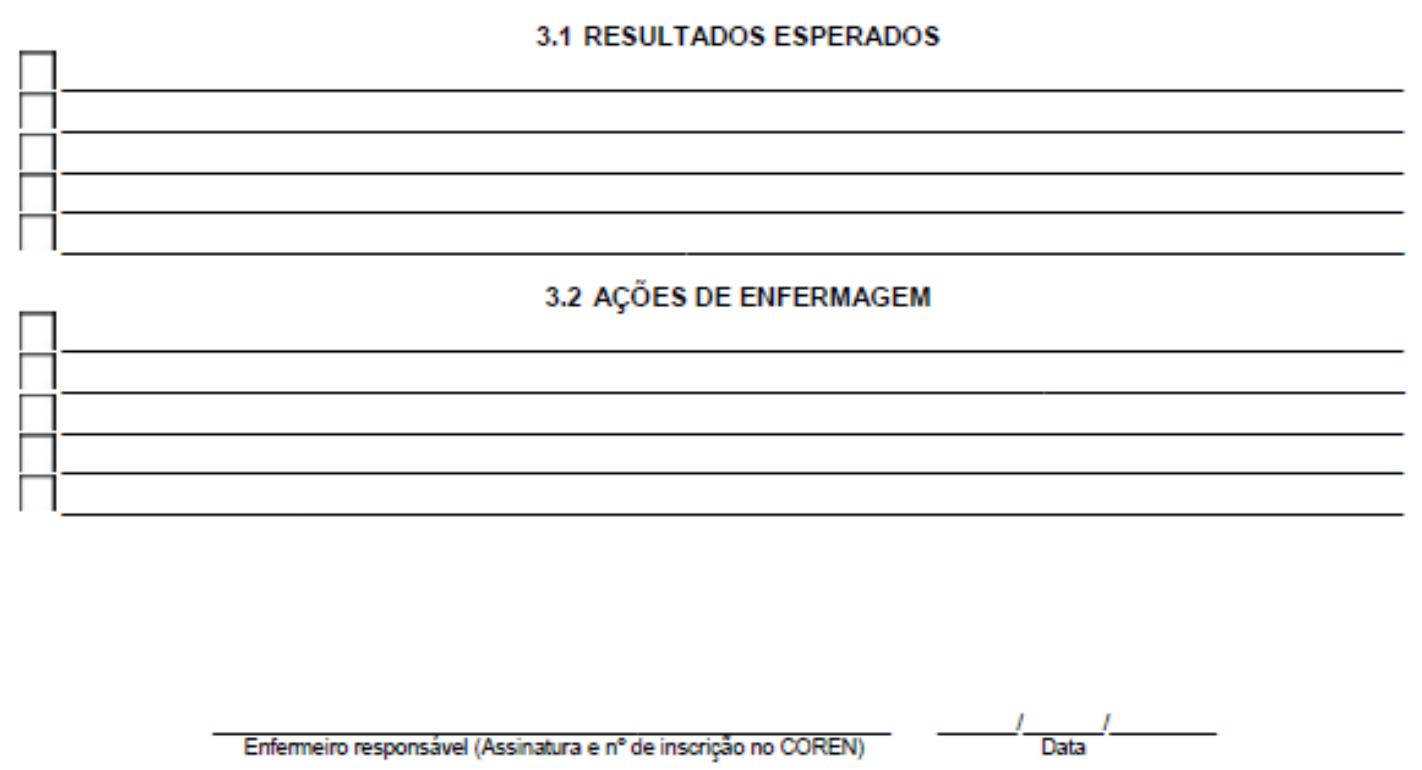
Editora Científica Chefe: Cristiane Cardoso de Paula

Editora associada: Graciela Dutra Sehnem

Fomento / Agradecimento: Este estudo recebeu auxílio financeiro oriundo do Programa de Iniciação Científica e Tecnológica da Universidade Estadual de Campinas, por meio de bolsa de iniciação científica por meio do Conselho Nacional de Desenvolvimento Científico e Tecnológico (CNPq).

\author{
Autor correspondente \\ Uiara Aline de Oliveira Kaizer \\ E-mail: uiara_oliveira@hotmail.com \\ Endereço: Tessália Vieira de Camargo, 126. Cidade Universitária. Faculdade de Enfermagem \\ CEP:13083887
}

\title{
Contribuições de Autoria
}

\section{1 - Maria Clara Moreira Matias}

concepção ou desenho do estudo/pesquisa, análise e/ou interpretação dos dados, revisão final com participação crítica e intelectual no manuscrito.

\section{2 - Uiara Aline de Oliveira Kaizer}

Revisão final com participação crítica e intelectual no manuscrito.

\section{3 - Thais Moreira São-João}

concepção ou desenho do estudo/pesquisa, análise e/ou interpretação dos dados, revisão final com participação crítica e intelectual no manuscrito.

\section{Como citar este artigo}

Matias MCM, Kaizer UAO, São-João TM. Consulta de enfermagem na Atenção Primária à Saúde: cuidado às pessoas com doenças crônicas cardiometabólicas. Rev. Enferm. UFSM. 2021 [Acesso em: Anos Mês Dia]; vol.11 e22: P1-22. DOI: https://doi.org/10.5902/2179769243719 